RENDICONTI DEL CIRCOLO MATEMATICO DI PALERMO

Serie II, Tomo XLIX (2000), pp. 371-380

\title{
NOTE ON HIT-AND-MISS TOPOLOGIES
}

\author{
LÁSZLÓ ZSILINSZKY
}

\begin{abstract}
This is a continuation of [19]. We characterize first and second countability of the general hit-and-miss hyperspace topology $\tau_{\Delta}^{+}$for weakly- $R_{0}$ base spaces. Further, metrizability of $\tau_{\Delta}^{+}$is characterized with no preliminary conditions on the base space and the generating family of closed sets and a new proof on uniformizability (i.e. complete regularity) of $\tau_{\Delta}^{+}$is given in this general setting, thus generalizing results of [3], [5] and [6].
\end{abstract}

\section{Introduction.}

Let $(X, \tau)$ be a topological space and $C L(X)$ be the nonempty closed subsets of $X$. Following [2], [3], [5], [16], [17], [19], [20], [21] we will continue to study hit-and-miss hyperspace topologies or $\Delta$-topologies on $C L(X)$, where $\Delta$ is a fixed subfamily of $C L(X)$. Two of the most studied hit-and-miss topologies are the Vietoris topology ([14], [13]) and the Fell topology ([7], [13], [17]). In a recent paper [5], Di Maio and Holá have found necessary and sufficient conditions for first and second countability, respectively of the $\Delta$-topology $\tau_{\Delta}^{+}$, if $X$ is $T_{1}$; more on countability axioms and quasi-uniformizability of $\tau_{\Delta}^{+}$was obtained by Holá and Levi in [9], where a characterization of metrizability of $\tau_{\Delta}^{+}$is also given for a $T_{1}$ base space $X$ and $\Delta$ containing the singletons. Moreover, in [3] (see also [2]),

1991 Mathematics Subject Classification. 54B20, Secondary 54D15, 54E35.

Key words and phrases. Hit-and-miss topologies, countability and separation axioms, metrizability, uniformizability. 
Beer and Tamaki characterized unifomizability of $\tau_{\Delta}^{+}$for a Hausdorff $X$ and $\Delta$ containing the singletons.

It is the purpose of this paper to show that quite similar characterizations hold with no preliminary conditions (or with much less restrictive conditions) on $X$ or $\Delta$, respectively. This is achieved by applying techniques and notions from [19] and a completely new approach is employed to characterize complete regularity of $\tau_{\Delta}^{+}$.

Note that a characterization of normality of $\tau_{\Delta}^{+}$is not known except for some special cases, like the Vietoris topology ([11], [18]) or the Fell topology ([10]); for some more general results on normality see [6].

\section{Notation and terminology.}

In the sequel $(X, \tau)$ will be a topological space and $C L(X)$ (resp. $K(X)$ ) will denote the nonempty closed (resp. nonempty closed compact) subsets of $X$. If $E \subset X$, then $\bar{E}$, int $E, E^{c}$ will stand for the closure, interior and complement of $E$, respectively in $X$. Put $E^{-}=\{A \in C L(X)$; $A \cap E \neq \emptyset\}, E^{+}=\{A \in C L(X) ; A \subset E\}$. In what follows, $\Delta$ will be a fixed but arbitrary nonempty subfamily of $C L(X)$ and for any $\Delta^{\prime} \subset \Delta$, denote by $\Sigma\left(\Delta^{\prime}\right)$ the set of all finite unions of members of $\Delta^{\prime}$. The hit-and-miss or $\Delta$-topology $\tau_{\Delta}^{+}$for $C L(X)$ has a base all sets of the form $\left(B^{c}\right)^{+} \cup \bigcap_{i=1}^{n} U_{i}^{-}$ where $B \in \Sigma(\Delta), U_{1}, \ldots, U_{n} \in \tau$ and $n \in \mathbb{N}$ (cf. [3], [17]); this basic element will be denoted by $\left(U_{1}, \ldots, U_{n}\right)_{B}^{+}$(cf; [20]). If $\Delta=C L(X)$, we obtain the familiar Vietoris topology $\tau_{V}$, if $\Delta=K(X)$, the Fell topology $\tau_{F}$.

In accordance with [3], $\Delta$ is said to be a Urysohn family provided whenever $A \in C L(X)$ and $B \in \Delta$ are disjoint, there exists $D \in \Sigma(\Delta)$ such that $B \subset$ int $D \subset D \subset A^{c}$. Denote by $\mathcal{F}_{\Delta}$ the class of all continuous functions $f: X \rightarrow[0,1]$ such that whenever inf $f<\alpha<\beta<\sup f$, there exists $D \in \Sigma(\Delta)$ with

$$
f^{-1}([0, \alpha]) \subset D \subset f^{-1}([0, \beta]),
$$

where $f^{-1}(M)$ stands for the preimage of $M \subset[0,1]$. For $f \in \mathcal{F}_{\Delta}$ denote by $m_{f}$ the infimal value functional on $C L(X)$ (cf. [3]) defined by

$$
m_{f}(A)=\inf \{f(x) ; x \in A\} \text { for all } A \in C L(X) .
$$


We will say that $X$ has property $P_{\Delta}$ provided whenever $A \in C L(X)$ and $x \in A^{c}$ there exists $D \in \Delta$ such that $D \subset A^{c}$ and $\overline{\{x\}} \cap D \neq \emptyset$ (see [19]). $X$ is called weakly- $R_{0}$ provided $X$ possesses property $P_{C L(X)}$ or equivalently provided every nonempty difference of $\tau$-open sets contains a nonempty closed subset of $X$ ([19]). Further, $X$ is an $R_{0}$-space if every open subset of $X$ contains the closure of each of its points ([4]).

We will say that $E \subset X$ is $c$-hemicompact if there exists an increasing sequence of members of $K(X) \cap C L(E)$ which is cofinal in $K(X) \cap C L(E)$. Notions not defined in the paper are used in accordance with [12] (e.g. regular does not include $T_{1}$ ).

\section{Main results.}

First we need some auxiliary material:

LEMMA 2.1. Let $X$ be weakly- $R_{0}, B, D \in \Sigma(\Delta)$ and $U_{1}, \ldots, U_{n}$, $V_{1}, \ldots, V_{m} \in \tau(m, n \in \mathbb{N})$. Then the following are equivalent:

(i) $\left(U_{1}, \ldots, U_{n}\right)_{B}^{+} \subset\left(V_{1}, \ldots, V_{m}\right)_{D}^{+}$;

(ii) $B^{c} \subset D^{c}$ and for every $1 \leq j \leq m$ there exists an $1 \leq i \leq n$ such that $U_{i} \cap B^{c} \subset V_{j} \cap D^{c}$.

Proof. Denote $\mathcal{U}=\left(U_{1}, \ldots, U_{n}\right)_{B}^{+}$and $\mathcal{V}=\left(V_{1}, \ldots, V_{m}\right)_{D}^{+}$. Suppose (i) and choose an $A \in \mathcal{U}$. If $B^{c} \backslash D^{c}$ is nonempty, then by the weak- $R_{0}$ property we can find a nonempty closed set $C \subset B^{c} \backslash D^{c}$. This implies that $A \cup C \in \mathcal{U} \backslash \mathcal{V}$, which contradicts (i), thus $B^{c} \subset D^{c}$. Further, if there exists a $1 \leq j \leq m$ such that for each $1 \leq i \leq n, \emptyset \neq U_{i} \cap B^{c} \backslash V_{j} \cap D^{c}$, then we can find a nonempty closed $A_{i} \subset U_{i} \cap B^{c} \backslash V_{j} \cap D^{c}$, but then $\bigcup_{i=1}^{m} A_{i} \in \mathcal{U} \backslash \mathcal{V}$, which is a contradiction again, so (ii) holds.

Conversely, suppose (ii) and pick an $A \in \mathcal{U}$. Then $A \subset B^{c} \subset D^{c}$. Further, for every $1 \leq j \leq m$ there is an $1 \leq i \leq n$ such that $U_{i} \cap B^{c} \subset V_{j} \cap D^{c}$, so $A \cap V_{j} \neq \emptyset$ since $A \cap U_{i} \neq \emptyset$. It means that $A \in \mathcal{V}$. $\square$

LEMMA 2.2. If $\left(C L(X), \tau_{\Delta}^{+}\right)$is first countable, then every $A \in C L(X)$ is separable.

Proof. The proof of Lemma 5.3 in [5] works in every topological space 
if point-closures are used instead of singletons.

We can now characterize first countability of the hit-and-miss topology for a weakly- $R_{0}$ base space $X$ (cf. [5], Theorem 5.4):

THEOREM 2.3. Let $X$ be a weakly- $R_{0}$ space. Then the following are equivalent:

(i) $\left(C l(X), \tau_{\Delta}^{+}\right)$is first countable;

(ii) $X$ is first countable, every closed set $A \subset X$ is separable and there exists a countable family $\Delta_{A} \subset \Delta$ such that whenever $B \in \Delta$ is disjoint to $A$, then $B \subset D \subset A^{c}$ for some $D \in \Sigma\left(\Delta_{A}\right)$.

Proof. The proof of Theorem 5.4 in [5] can be abopted if Lemma 2.1, Lemma 2.2 and point-closures are used insetad of singletons. In the implication (i) $\Rightarrow$ (ii) only the proof of first countability of $X$ needs some comments. Let $x \in X$ and put $A_{x}=\overline{\{x\}}$. In view of (i) there exist countable families $\Delta_{x} \subset \Delta$ and $\tau_{x} \subset \tau$ such that $\mathcal{B}_{x}=\left\{\left(U_{1}, \ldots, U_{n}\right)_{B}^{+} ; B \in \Sigma\left(\Delta_{x}\right)\right.$, $\left.U_{1}, \ldots, U_{n} \in \tau_{x}, n \in \mathbb{N}\right\}$ forms a countable local base at $A_{x}$ in $\tau_{\Delta}^{+}$. Choose any $\tau$-open neighborhood $U$ of $x$. Then $\mathcal{U}=\left(U_{1}, \ldots, U_{n}\right)_{B}^{+} \subset U^{-}$for some $\mathcal{U} \in \mathcal{B}_{x}$, thus by Lemma $2.1, B^{c} \cap U_{i} \subset U$ for an $1 \leq i \leq n$ and clearly $x \in B^{c} \cap U_{i}$. It means that $\left\{B^{c} \cap U ; B \in \Sigma\left(\Delta_{x}\right), U \in \tau_{x}\right\}$ is a countable local base at $x$.

As for second countability of the hit-and-miss topology we have (cf. [5], Theorem 5.13):

THEOREM 2.4. Let $X$ be a weakly- $R_{0}$ space. Then the following are equivalent:

(i) $\left(C L(X), \tau_{\Delta}^{+}\right)$is second countable;

(ii) $X$ is second countable and there is a countable family $\Delta^{\prime} \subset \Delta$ such that whenever $B \in \Delta$ and $A \in C L(X)$ are disjoint, then $B \subset D \subset A^{c}$ for some $D \in \Sigma\left(\Delta^{\prime}\right)$.

Proof. From (i) we get countable families $\Delta^{\prime} \subset \Delta, \tau^{\prime} \subset \tau$ such that

$$
\left\{\left(U_{1}, \ldots, U_{n}\right)_{B}^{+} ; B \in \Sigma\left(\Delta^{\prime}\right), U_{1}, \ldots, U_{n} \in \tau^{\prime}, n \in \mathbb{N}\right\}
$$


forms a countable base of $\tau_{\Delta}^{+}$. Then $\left\{B^{c} \cap U ; B \in \Sigma\left(\Delta^{\prime}\right), U \in \tau^{\prime}\right\}$ is a countable base for $X$, which easily follows by Lemma 2.1 . The rest of the proof is analoguous to that of Theorem 5.13 in [5].

It is shown in [19] that regularity and $T_{3}$-ness of the Vietoris topology are equivalent. We show that it is a general feature of hit-and-miss topologies. First we need the following:

LEMMA 2.5. The functional $m_{f}: C L(X) \rightarrow[0,1]$ is $\tau_{\Delta}^{+}$-continuous for all $f \in \mathcal{F}_{\Delta}$.

Proof. Choose $f \in \mathcal{F}_{\Delta}$. Let inf $f<\alpha<\beta<\sup f$ and $E \in$ $m_{f}^{-1}((\alpha, \beta))$. Then $\alpha<\inf \{f(x) ; x \in E\}<\beta$, thus $E \cap f^{-1}((\alpha, \beta)) \neq \emptyset$ and for any $0<\varepsilon<m_{f}(E)-\alpha$ we have $f^{-1}([0, \alpha+\varepsilon]) \subset E^{c}$. Since $f \in \mathcal{F}_{\Delta}$ we can find a $D \in \Sigma(\Delta)$ such that

$$
f^{-1}([0, \alpha+\varepsilon / 2]) \subset D \subset f^{-1}([0, \alpha+\varepsilon]),
$$

whence $E \subset D^{c}$. Then $E \in\left(D^{c}\right)^{+} \cap\left(f^{-1}((\alpha, \beta))\right)^{-} \subset m_{f}^{-1}((\alpha, \beta))$.

The following theorem is proved in [3] (Theorem 3.6) for a $T_{2}$ base space and with $\Delta$ containing the singletons. Here we present a different proof in the completely general setting:

THEOREM 2.6. The following are equivalent

(i) $\left(C L(X), \tau_{\Delta}^{+}\right)$is a Tychonoff space;

(ii) $\left(C L(X), \tau_{\Delta}^{+}\right)$is completely regular;

iii) $\left(C L(X), \tau_{\Delta}^{+}\right)$is a $T_{3}$-space;

(iv) $\left(C L(X), \tau_{\Delta}^{+}\right)$is regular;

(v) $X$ has property $P_{\Delta}$ and $\Delta$ is a Urysohn family.

Proof. (v) $\Rightarrow$ (i) In view of Theorem 1 in [19] it suffices to prove that the hyperspace is completely regular. An argument similar to that of in Lemma 3.1 of [3] yields for all $D \in \Delta$ and disjoint $A \in C L(X)$ an $f \in \mathcal{F}_{\Delta}$ such that $f(D)=0$ and $f(A)=1$. Let $A \in C L(X)$ and $\mathcal{U}=\left(U_{1}, \ldots, U_{n}\right)_{B}^{+}$be a $\tau_{\Delta}^{+}$-neighborhood of $A$, where $B \in \Sigma(\Delta), U_{1}, \ldots, U_{n} \in \tau$ and $n \in \mathbb{N}$. 
Then $A \subset B^{c}$ and $A \cap U_{i} \neq \emptyset$ for all $1 \leq i \leq n$. In virtue of the preceding considerations there exist functions $f_{0}, f_{1}, \ldots, f_{n} \in \mathcal{F}_{\Delta}$ such that

$$
\begin{aligned}
& f_{0}(B)=\{0\} \text { and } f_{0}(A)=\{1\}, \\
f_{i}\left(E_{i}\right)= & \{0\} \text { and } f_{i}\left(U_{i}^{c}\right)=\{1\} \text { for each } 1 \leq i \leq n .
\end{aligned}
$$

The by Lemma $2.5, m_{f_{0}}, m_{f_{1}}, \ldots, m_{f_{n}}$ are $\tau_{\Delta}^{+}$-continuous on $C L(X)$ so $F=\max \left\{1-m_{f_{0}}, m_{f_{1}}, \ldots, m_{f_{n}}\right\}$ is $\tau_{\Delta}^{+}$-continuous as well. Clearly $1-m_{f_{0}}(A)=m_{f_{1}}(A)=\cdots=m_{f_{n}}(A)=0$ so $F(A)=0$. Further if $E \notin \mathcal{U}$ then either $E \cap B \neq \emptyset$ or $E \subset U_{i}^{c}$ for some $1 \leq i \leq n$. In the first case $1-m_{f_{0}}(E)=1$, whence $F(E)=1$ and in the second case $m_{f_{i}}(E)=1$, so $F(E)=1$ again.

All the remaining implications follows from Theorem 3 in [19], if regularity of the hyperspace forces $X$ to have property $P_{\Delta}$. Indeed, if $\left(C L(X), \tau_{\Delta}^{+}\right)$is regular then it is also $R_{0}$, further the hit-and-miss topology is always $T_{0}$ (see [16]) so it is a $T_{1}$-space (cf. [4], Corollary), which completes the proof by Theorem 1 in [19].

If $X$ is a Hausdorff space and $\Delta$ contains the singletons then $X$ clearly possesses property $P_{\Delta}$. Thus the following corollary generalises Theorem 3.6 of [3]:

COROLLARY 2.7. $\left(C L(X), \tau_{\Delta}^{+}\right)$is uniformizable if and only if $X$ possesses property $P_{\Delta}$ and $\Delta$ is a Urysohn family.

Finally we turn to characterizing metrizability of the hit-and-miss topology:

THEOREM 2.8. The following are equivalent:

(i) $\left(C L(X), \tau_{\Delta}^{+}\right)$is metrizable;

(ii) $\left(C L(X), \tau_{\Delta}^{+}\right)$is pseudo-metrizable;

(iii) $\left(C L(X), \tau_{\Delta}^{+}\right)$is second countable and regular,

(iv) $X$ possesses property $P_{\Delta}$ and there exists a countable family $\Delta^{\prime} \subset \Delta$ such that whenever $B \in \Delta$ and $A \in C L(X)$ are disjoint there is a $D \in \Sigma\left(\Delta^{\prime}\right)$ with $B \subset$ int $D \subset D \subset A^{c}$. 
Proof. The equivalence (i) $\Leftrightarrow$ (ii) follows from Theorem 2.8. For (i) $\Rightarrow$ (iii) see Proposition $5.18(1) \Rightarrow$ (2) in [5], further our Lemma 2.2 and use point-closures instead of singletons. Now suppose (iii). Regularity of $\left(C L(X), \tau_{\Delta}^{+}\right)$implies by Lemma 2(ii) of [19] that $X$ is weakly- $R_{0}$, so our Theorem 2.4 and Theorem 2.6 implies (iv) similarly as in [5] (Theorem $5.19(1) \Rightarrow(2))$. Finally, if we assume (iv) then according to Theorem 2.6, $\left(C L(X), \tau_{\Delta}^{+}\right)$is a $T_{3}$-space, consequently by Lemma 2(ii) of [19], $X$ is weakly- $R_{0}$ so if $X$ was second countable then in view of Theorem 2.4 the $\Delta$-topology would be second countable and the Urysohn Metrization Theorem would yield (i). Hence, it remains to justify that the countable family $\mathcal{B}=\left\{\right.$ int $\left.D ; D \in \Sigma\left(\Delta^{\prime}\right)\right\}$ is a base for $(X, \tau)$. Indeed, if $U$ is a nonempty $\tau$-open set and $x \in U$, then by property $P_{\Delta}$ there exists $B \in \Delta$ with $B \subset U$ and $B \cap \overline{\{x\}} \neq \emptyset$. In virtue of the second condition of (iv) we can find $D \in \Sigma\left(\Delta^{\prime}\right)$ such that $B \subset$ int $D \subset D \subset U$ (we can assume that $U \neq X)$. Then $x \in$ int $D \subset U$.

Remark 2.9. It is inferable from the proof of the preceding theorem that metrizability of $\left(C L(X), \tau_{\Delta}^{+}\right)$always forces second countability on $X$.

\section{Applications.}

In view of our preceding theorems we have:

THEOREM 3.1. (cf. [5], Theorem 5.5) Let $X$ be weakly- $R_{0}$. Then the following are equivalent:

(i) $\left(C L(X), \tau_{V}\right)$ is first countable;

(ii) every closed subset of $X$ is separable and has a countable base of neighborhoods.

THEOREM 3.2. (cf. [5], Theorem 5.6) Let $X$ be weakly- $R_{0}$. Then the following are equivalent:

(i) $\left(C L(X), \tau_{F}\right)$ s first countable;

(ii) $X$ is first countable every closed set is separable and every proper open subset is c-hemicompact.

Proof. (i) $\Rightarrow$ (ii) The proof of hemicompactness of proper open subsets 
of $X$ in [1], Lemma 3.1 is feasible also in our case if closed compact sets are used instead of compact sets and point-closures instead of singletons. Further see our Theorem 2.3. In (ii) $\Rightarrow$ (i) the proof of [5], Theorem $5.6(2) \Rightarrow(1)$ is applicable (using $c$-hemicompactness instead of hemicompactness) along with our Theorem 2.3.

THEOREM 3.3. (cf. [19]; Theorem 4) The following are equivalent:

(i) $\left(C L(X), \tau_{V}\right)$ is a Tychonoff space;

(ii) $\left(C L(X), \tau_{V}\right)$ is completely regular;

(iii) $\left(C L(X), \tau_{V}\right)$ is a $T_{3}$-space;

(iv) $\left(C L(X), \tau_{V}\right)$ is regular;

(v) $\left(C L(X), \tau_{V}\right)$ is uniformizable;

(vi) $X$ is normal and $R_{0}$.

THEOREM 3.4. The following are equivalent:

(i) $\left(C L(X), \tau_{F}\right)$ is a Tychonoff space;

(ii) $\left(C L(X), \tau_{F}\right)$ is regular;

(iii) $\left(C L(X), \tau_{F}\right)$ is a Hausdorff space;

(iv) $\left(C L(X), \tau_{F}\right)$ is uniformizable;

(v) $X$ is a locally compact, regular space.

Proof. Cf. [17] (Folgerung (a), p. 162) and Theorem 2 of [19].

THEOREM 3.5. (cf. [14], Theorem 4.9.7) The following are equivalent:

(i) $\left(C L(X), \tau_{V}\right)$ is metrizable;

(ii) $X$ is compact and pseudo-metrizable.

Proof. (i) $\Rightarrow$ (ii) Suppose that $\left(C L(X), \tau_{V}\right)$ is metrizable. Denote by $\tilde{X}$ the quotient space of $X$ induced by identification of points with common closure in $X$. Then in view of Theorem 3 in [15], $\left(C L(\tilde{X}), \tilde{\tau}_{V}\right)$ is homeomorphic to $\left(C L(X), \tau_{V}\right)$, where $\tilde{\tau}_{V}$ is the Vietoris topology on $C L(\tilde{X})$, 
consequently it is also metrizable. Further, $\left(C L(X), \tau_{V}\right)$ is a Hausdorff space so by Theorem 2 of [19], $X$ is regular and hence $R_{0}$ as well. Accordingly $\tilde{X}$ is a $T_{1}$-space, thus by Theorem 4.9 .7 of [14], $\tilde{X}$ is compact, which implies compactness of $X$ (cf. [15], Theorem 4). Now $X$ is second countable by Remark 2.9, further it is regular, thus $X$ is pseudo-metrizable (see [8], p. 167, Exercise 3).

(ii) $\Rightarrow$ (i) Observe that a pseudo-metrizable space is $R_{0}$, hence possesses property $P_{C L(X)}$ (i.e. weak $R_{0}$-ness). Further by Lemma 2.2, $X$ is a separable (pseudo-metrizable) space, accordingly second countable as well, which together with compactness and regularity of $X$ easily yields the second condition of Theorem 2.8 (iv) for $\Delta=C L(X)$.

THEOREM 3.6. (cf. [1], Theorem 3.4) The following are equivalent:

(i) $\left(C L(X), \tau_{F}\right)$ is metrizable;

(ii) $X$ is locally compact, regular and second countable.

Proof. (i) $\Rightarrow$ (ii) According to Remark 2.9, $X$ is second countable and in virtue of Theorem $3.4, X$ is locally compact and regular.

(ii) $\Rightarrow$ (i) By local compactness plus regularity of $X, K(X)$ forms a base of neighborhoods for closed compact subsets of $X$ ([12], p. 146, Theorem $18)$. Further, second countability of $X$ yields a countable subfamily of $K(X)$ which forms also a base of neighboroods for members of $K(X)$, thus the second condition of Theorem 2.8 (iv) is fulfilled for $\Delta=K(X)$. Finally, local compactness and regularity of $X$ evidently imply property $P_{K(X)}$, thus Theorem 2.8 applies.

\section{REFERENCES}

[1] Beer G., On the Fell Topology, Set-Valued Analysis, 1 (1993), 69-80.

[2] Beer G., Topologies on Closed and Closed Convex Sets, Kluwer, Dordrecht, 1993.

[3] Beer G., Tamaki R., The Infimal Value Functional and the Uniformization of Hitand-Miss Hyperspace Topologies, Proc. Amer. Math. Soc., 122 (1994), 601-612.

[4] Davis A., Indexed systems of neighborhoods for general topological spaces, Amer. Math. Monthly, 68 (1961), 886-893.

[5] Di Maio G., Holá L', On hit-and-miss topologies, Rend. Acc. Sc. Fis. Mat. Napoli, 62 (1995), 103-124. 
[6] Di Maio G., Holá L', Meccariello E., Notes on hit-and-miss topologies, Rostocker Math. Kolloq., to appear.

[7] Fell J., A Hausdorff topology for the closed subsets of a locally compact nonHausdorff space, Proc. Amer. Math. Soc., 13 (1962), 472-476.

[8] Gaal S. A., Point Set Topology, Academic Press, New York, 1964.

[9] Holá L'., Levi S., Decomposition properties of hyperspaces topologies, Set-Valued Anal., 5 (1997), 309-321.

[10] Holá L'., Levi S., Pelant J., Normality and Paracompactness of the Fell topology, Proc. Amer. Math., Soc., 127 (1999), 2193-2197.

[11] Keesling J., On the equivalence of normality and compactness in hyperspaces, Pacific J. Math., 33 (1970), 657-667.

[12] Kelley J. L., General Topology, Springer-Verlag, New York, 1975.

[13] Klein E., Thompson A., Theory of Correspondences, Wiley, New York, 1975.

[14] Michael E., Topologies on spaces of subsets, Trans. Amer. Math. Soc., 71 (1951), 152-182.

[15] Mrševič M., Some proeprties of the space $2^{X}$ of a topological $R_{0}$-space, Uspechi Mat. Nauk, 34 n. 6 (210) (1979), 166-170 (Russian).

[16] Poppe H., Eine Bemerkung über Trennungsaxiome in Räumen von abgeschlossenen Teilmengen topologisher Räume, Arch. Math., 16 (1965), 197-199.

[17] Poppe H., Einige Bemerkungen über den Raum der abgeschlossenen Mengen, Fund. Math., 59 (1966), 159-169.

[18] Veličko N. H., On the space of closed subsets, Sibirsk. Math. Z. 16 (1975), 627-629. (Russian; English translation: Siberian Math. J., 16 (1975), 484-486).

[19] Zsilinszky L., On separation axioms in hyperspaces, Rend. Circ. Matem. Palermo, 45 (1996), 75-83.

[20] Zsilinszky L., Baire space and hyperspace topologies, Proc. Amer. Math. Soc., 124 (1996), 3175-3184.

[21] Zsilinszky L., Baire spaces and weak topologies generated by gap and excess functionals, Math. Slovaca, 49 (1999), 357-366.

Pervenuto il 10 luglio 1996

In forma modificata il 2 luglio 1999.

Department of Mathematics and Computer Science University of North Carolina

at Pembroke
Pembroke, NC 28372 - USA

E-mail address: laszlo@nat.uncp.edu 